\title{
Iteratively Detected Three-Stage Multi-Dimensional Sphere Packing Modulation Aided Multi-Functional MIMO
}

\author{
Mohammed El-Hajjar, Osamah Alamri and Lajos Hanzo \\ School of Electronics and Computer Science, University of Southampton, SO17 1BJ, UK. \\ Email: $\{$ meh05r, ora02r, 1h\}@ecs.soton.ac.uk, \\ http://www-mobile.ecs.soton.ac.uk
}

\begin{abstract}
This paper presents a novel multi-functional MultipleInput Multiple-Output (MIMO) scheme that combines the benefits of the Vertical Bell Labs Layered Space-Time (V-BLAST) scheme, of SpaceTime Codes (STC) as well as of beamforming. To further enhance the attainable system performance and to maximise the coding advantage of the proposed transmission scheme, the system is also combined with multi-dimensional Sphere Packing (SP) modulation. Additionally, further system performance improvements can be attained by serially concatenated convolutional coding combined with a Unity-Rate Code (URC) employed as an inner code. Then, at the receiver side, iterative decoding is invoked by exchanging extrinsic information between the three constituent decoders, i.e. the outer convolutional code's decoder, the inner URC's decoder as well as the SP demapper. Moreover, the convergence behaviour of the proposed scheme is evaluated with the aid of both three-dimensional (3D) and two-dimensional (2D) Extrinsic Information Transfer (EXIT) charts. Finally, we quantify the maximum achievable rate of the system based on EXIT charts and demonstrate that the iterative-detection-aided system is capable of operating within $0.6 \mathrm{~dB}$ from the maximum achievable rate limit. Explicitly, the proposed iteratively detected three-stage LSSTC-SP scheme is capable of attaining at least $4.8 \mathrm{~dB}$ gain at a BER of $10^{-5}$ over the conventional iterativedetection aided two-stage scheme, where the extrinsic information is limited to the SP demapper and the outer code's decoder.
\end{abstract}

\section{INTRODUCTION}

Information theoretic studies $[1,2]$ have revealed that a MultipleInput Multiple-Output (MIMO) system attains a higher capacity than its single-input single-output counterpart. The Vertical Bell Labs Layered Space-Time (V-BLAST) scheme proposed in [3] is capable of providing a substantial increase of a specific user's effective spectral efficiency. On the other hand, Space-Time Block Codes (STBC), that were introduced in $[4,5]$, constitute a powerful transmit diversity scheme. Thus, it was proposed in [6] to combine the benefits of these two techniques for the sake of providing both antenna diversity as well as multiplexing gains. This hybrid scheme was improved in [7] by optimising the decoding order of the different antenna layers. Furthermore, in order to achieve additional performance gains, beamforming [8] has been combined with STBC to attain a higher Signal-to-Noise Ratio (SNR) gain [9]. Additionally, it was proposed in [10] to combine the benefits of V-BLAST, STBC and beamforming in order to design a system having a high multiplexing gain, a high diversity gain as well as a beamformer gain. The scheme proposed in [10] is referred to as a Layered Steered Space-Time Code (LSSTC).

Moreover, Sphere Packing (SP) was combined with the concept of orthogonal transmit diversity designs in [11] in order to maximise the achievable coding advantage, where $\mathrm{Su}$ et al. demonstrated in [11] that the proposed SP aided STBC scheme was capable of outperforming the conventional orthogonal design based STBC schemes of $[4,5]$. Furthermore, Alamri et al. [12] modified the SP

The financial support of Vodafone under the auspices of the Dorothy Hodgkin Postgraduate Award and that of the European Union within the Newcom and Pheonix projects, and the support of EPSRC, UK is gratefully acknowledged. demapper of [11] for the sake of accepting the a priori information passed to it from the channel decoder as extrinsic information.

The turbo principle [13] was considered in [14] for iterative soft demapping in the context of multilevel modulation schemes combined with channel decoding, where a soft symbol-to-bit demapper was used between the multilevel demodulator and the binary channel decoder. Moreover, in [15] it was shown that a recursive inner code is needed in order to avoid the formation of a Bit-Error Ratio (BER) floor. Additionally, in [16] unity-rate inner codes were employed in iteratively detected, bandwidth as well as power limited systems having stringent BER requirements. Furthermore, Extrinsic Information Transfer (EXIT) charts [17] have been proposed for studying the convergence behaviour of iterative decoding by describing the flow of extrinsic information through the soft-in soft-out constituent decoders. The concept of EXIT chart analysis has been extended to three-stage concatenated systems in [18-20].

The novelty and rationale of the proposed system can be summarised as follows:

1) We employ a MIMO scheme that amalgamates the merits of $V$-BLAST, STC and beamforming for the sake of achieving a high multiplexing gain, a high diversity gain as well as beamformer gain. Additionally, the system is combined with multidimensional SP modulation, which is capable of maximising the coding advantage of the transmission scheme by jointly designing and detecting the sphere-packed space-time symbols.

2) The optimum bit-to-SP-symbol mapper is designed using an EXIT-chart based procedure, which allows us to achieve diverse design objectives. For example, we can design a system having the lowest possible turbo-cliff-SNR, but tolerating the formation of an error floor at low BERs. Alternatively, we can design a system having no error floor, but having a slightly higher turbocliff-SNR.

3) We propose a novel technique for quantifying the maximum achievable rate of the system using EXIT charts.

4) We propose a near-capacity iteratively detected three-stage LSSTC-SP receiver structure, where iterative detection is carried out between an outer code's Decoder I, an intermediate code's Decoder II and an LSSTC-SP demapper. The intermediate code employed is a Unity-Rate Code (URC), which is capable of completely eliminating the system's error-floor as well as of operating at the lowest possible turbo-cliff SNR without significantly increasing the associated complexity or the interleaver delay. Furthermore, the proposed three-stage system is capable of operating within $0.6 \mathrm{~dB}$ from the maximum achievable rate limit obtained using EXIT charts.

5) The proposed iteratively detected three-stage system is compared to an iteratively detected two-stage system employing iterations between the outer code's decoder and the SP demapper. The three-stage system has been found to outperform the two-stage system by at least $4.8 \mathrm{~dB}$ at a BER of $10^{-5}$. 


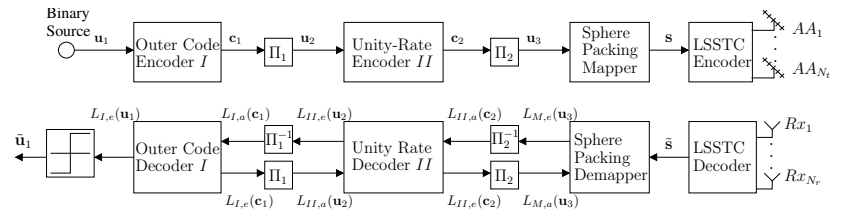

Fig. 1. Block diagram of the iteratively detected three-stage LSSTC-SP scheme.

The rest of the paper is organised as follows. In Section II an overview of the iterative-detection-aided multi-dimensional modulation assisted multi-functional MIMO scheme is presented. Section III outlines the rationale of combining LSSTC with multi-dimensional SP modulation. In Section IV the three-stage iterative detection process is detailed together with the associated 3D EXIT charts and their $2 \mathrm{D}$ projection. Section $\mathrm{V}$ presents our performance results, followed by our conclusions in Section VI.

\section{SYSTEM OVERVIEW}

The block diagram of the proposed iteratively detected three-stage LSSTC-SP scheme is illustrated in Figure 1. A detailed discussion of the LSSTC encoding and decoding processes is presented in [10]. The antenna architecture employed in the LSSTC scheme has $N_{t}$ transmit Antenna Arrays (AA) spaced sufficiently far apart in order to experience independent fading and hence to achieve transmit diversity. The $L_{A A}$ number of elements of each of the AAs are spaced at a distance of $d=\lambda / 2$ for the sake of achieving a beamformer gain, where $\lambda$ represents the carrier's wavelength. Furthermore, the receiver is equipped with $N_{r} \geqslant N_{t}$ antennas. According to [10], a block of $B$ input information symbols is serial-to-parallel converted to $K$ groups of symbol streams of length $B_{1}, B_{2}, \cdots, B_{K}$, where we have $B_{1}+B_{2}+\cdots+B_{K}=B$. Each group of $B_{k}$ symbols, $k \in[1, K]$, is then encoded by a component space-time code $\mathrm{STC}_{k}$ associated with $m_{k}$ transmit AAs, where $m_{1}+m_{2}+\cdots+m_{K}=N_{t}$. More specifically, here we consider a system employing $N_{t}=4$ transmit antennas, $N_{r}=4$ receive antennas, $L_{A A}=4$ elements per AA as well as $K=2$ twin-antenna-aided STBC layers.

In this contribution, we consider transmissions over a temporally correlated narrowband Rayleigh fading channel associated with a normalised Doppler frequency of $f_{D}=f_{d} T_{s}=0.01$, with $f_{d}$ being the Doppler frequency and $T_{s}$ the symbol duration, while the spatial channel coefficients are independent. The complex Additive White Gaussian Noise (AWGN) of $n=n_{I}+j n_{Q}$ contaminates the received signal, where $n_{I}$ and $n_{Q}$ are two independent zero-mean Gaussian random variables having a variance of $N_{0} / 2$ per dimension.

According to Figure 1, the transmitted source bits $\mathbf{u}_{1}$ are encoded by a 1/2-rate Recursive Systematic Convolutional (RSC) code and then interleaved by a random bit interleaver $\Pi_{1}$. After channel interleaving the symbols are precoded by a URC followed by another random interleaving process $\Pi_{2}$. Then, the interleaved bits are mapped to their corresponding SP symbols in the SP mapper of Figure 1. The SP mapper maps $B_{s p}$ channel-coded bits $\mathbf{b}=b_{0}, \ldots, b_{B_{s p}-1} \in\{0,1\}$ to a multi-dimensional SP symbol s. Subsequently, the SP modulated symbols are serial-to-parallel converted to two substreams, so that each substream is transmitted using STBC.

At the receiver side, as shown in Figure 1, the received complexvalued symbols are first decoded by the LSSTC decoder in order to produce the received SP soft-symbol estimate $\tilde{\mathbf{s}}$. Then, iterative detection is carried out between the SP demapper, the APPbased Soft-In/Soft-Out (SISO) URC Decoder II and the APP-based SISO Decoder I, where extrinsic information is exchanged between the three constituent demapper/decoder modules. More specifically, $L \cdot, a(\cdot)$ in Figure 1 represents the a priori information, expressed in terms of the log-likelihood ratios (LLRs) of the corresponding bits, whereas $L_{., e}(\cdot)$ represents the extrinsic LLRs of the corresponding bits. The iterative process is performed for a number of consecutive iterations. During the last iteration, only the LLR values $L_{I, e}\left(\mathbf{u}_{\mathbf{1}}\right)$ of the original data information bits $\mathbf{u}_{1}$ are required, which are passed to a hard decision decoder in order to determine the estimated transmitted source bits $\tilde{\mathbf{u}}_{1}$, as shown in Figure 1.

\section{Layered Steered Space-Time Codes Aided Sphere PaCKing Modulation}

The LSSTC decoded signal represents a scaled version of the transmitted signal corrupted by AWGN. This observation implies that the diversity product ${ }^{1}$ of the LSSTC scheme is determined by the Minimum Euclidean Distance (MED) of all legitimate transmitted vectors. Hence, in order to maximise the achievable diversity product, it was proposed in [11] to use SP schemes that maximise the MED of the transmitted signal vectors. While in Alamouti's now classic design [4] no attempt is made to jointly shape the modulated signals of the two antennas and two time-slots, in our approach we jointly design the legitimate two-component complex-valued vectors $\left(x_{1}, x_{2}\right)_{k}$ transmitted from layer $k, k \in[0,1]$, so that they are represented by a single phasor point selected from a SP constellation corresponding to a four-dimensional real-valued lattice having the best known MED in the four-dimensional real-valued space $\mathbb{R}^{4}$.

To elaborate a little further, according to [10] $x_{1}$ and $x_{2}$ represent independent conventional PSK modulated symbols transmitted from the first and second transmit AA and no effort is made to jointly design a signal constellation for the various combinations of $x_{1}$ and $x_{2}$. By contrast, in the case of SP, these symbols are designed jointly in order to further increase the attainable diversity product. Assuming that there are $L$ legitimate vectors $\left(x_{l, 1}, x_{l, 2}\right), l=0,1, \ldots, L-$ 1 , where $L$ represents the number of SP modulated symbols, the transmitter then has to choose the modulated signal from the set of legitimate symbols to be transmitted over the two AAs in each layer.

In the four-dimensional real-valued Euclidean space $\mathbb{R}^{4}$, the lattice $D_{4}$ is defined as a SP constellation having the best MED from all other $(L-1)$ legitimate four-component constellation points in $\mathbb{R}^{4}$ [21]. Assuming that $\mathbf{S}=\left\{\mathbf{s}^{l}=\left[a_{l, 1}, a_{l, 2}, a_{l, 3}, a_{l, 4}\right] \in \mathbb{R}^{4}\right.$ : $0 \leq l \leq L-1\}$ constitutes a set of $L$ legitimate constellation points from the lattice $D_{4}$ having a total energy of $E \triangleq \sum_{l=0}^{L-1}\left(\left|a_{l, 1}\right|^{2}+\right.$ $\left.\left|a_{l, 2}\right|^{2}+\left|a_{l, 3}\right|^{2}+\left|a_{l, 4}\right|^{2}\right)$, upon introducing the notation

$$
\mathbf{C}_{l}=\left\{x_{l, 1}, x_{l, 2}\right\}=\left\{a_{l, 1}+j a_{l, 2}, a_{l, 3}+j a_{l, 4}\right\},
$$

we have a set of constellation symbols, $\left\{\mathbf{C}_{l}: 0 \leq l \leq L-1\right\}$, leading to the design of LSSTC signals, whose diversity product is determined by the MED of the set of $L$ legitimate constellation points in $\mathbf{S}$.

\section{Iterative Decoding AND EXIT Chart ANALysis}

As shown in Figure 1, the received complex-valued symbols are first decoded by the LSSTC decoder in order to produce the received SP symbol estimates $\tilde{\mathbf{s}}$, where each SP symbol represents a block of $B_{s p}$ coded bits, as described in Section II. Then, iterative detection is carried out between the SP demapper, the SISO URC Decoder II and the SISO outer Decoder I, where extrinsic information is exchanged between the three constituent demapper/decoder modules.

\footnotetext{
${ }^{1}$ The diversity product or coding advantage is defined as the estimated gain over an uncoded system having the same diversity order as the coded system [11].
} 


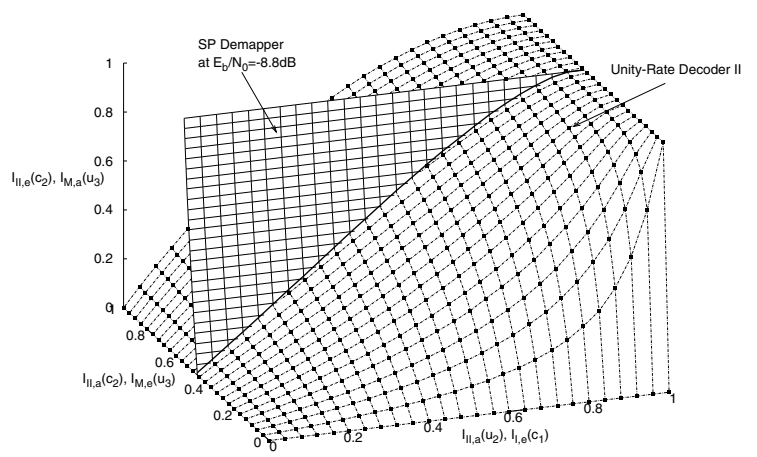

Fig. 2. 3D EXIT chart of the URC Decoder II and the SP demapper at $E_{b} / N_{0}=-8.8 \mathrm{~dB}$.

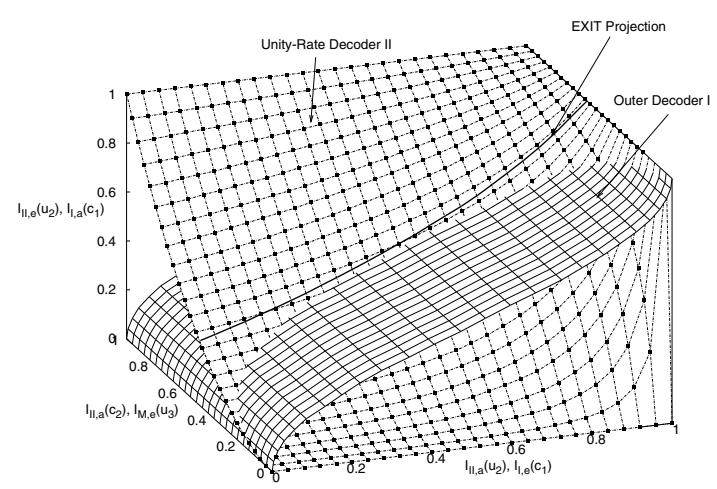

Fig. 3. 3D EXIT chart of the URC Decoder II and the RSC Decoder I with projection from Figure 2.

\section{A. 3D EXIT Charts}

As seen in Figure 1, the input of Decoder II is constituted by the a priori input $L_{I I, a}\left(\mathbf{c}_{2}\right)$ and the a priori input $L_{I I, a}\left(\mathbf{u}_{2}\right)$ after appropriately ordering the data provided by the SP demapper and Decoder I, respectively. Therefore, the EXIT characteristics of Decoder II can be described by the following two EXIT functions [17, 20]:

$$
\begin{aligned}
I_{I I, e}\left(\mathbf{c}_{2}\right) & =T_{I I, c_{2}}\left[I_{I I, a}\left(\mathbf{u}_{2}\right), I_{I I, a}\left(\mathbf{c}_{2}\right)\right] \\
I_{I I, e}\left(\mathbf{u}_{2}\right) & =T_{I I, u_{2}}\left[I_{I I, a}\left(\mathbf{u}_{2}\right), I_{I I, a}\left(\mathbf{c}_{2}\right)\right]
\end{aligned}
$$

which are illustrated by the $3 \mathrm{D}$ surfaces drawn in dotted lines in Figures 2 and 3, respectively. On the other hand, the EXIT characteristics of the SP demapper as well as those of Decoder I are each dependent on a single a priori input, namely on $L_{M, a}\left(\mathbf{u}_{3}\right)$ and $L_{I, a}\left(\mathbf{c}_{1}\right)$, respectively, both of which are provided by the URC Decoder II after appropriately ordering the bits, as seen in Figure 1. The EXIT characteristics of the SP demapper are also dependent on the $E_{b} / N_{0}$ value. Consequently, the corresponding EXIT functions for the SP demapper and Decoder I may be written, respectively, as

$$
\begin{aligned}
I_{M, e}\left(\mathbf{u}_{3}\right) & =T_{M, u_{3}}\left[I_{M, a}\left(\mathbf{u}_{3}\right), E_{b} / N_{0}\right] \\
I_{I, e}\left(\mathbf{c}_{1}\right) & =T_{I, c_{1}}\left[I_{I, a}\left(\mathbf{c}_{1}\right)\right],
\end{aligned}
$$

which are illustrated by the $3 \mathrm{D}$ surfaces drawn in solid lines in Figures 2 and 3, respectively.

Equations (2) to (5) can be represented with the aid of two 3D EXIT charts. More specifically, the 3D EXIT chart of Figure 2 is used to plot Equation (2) and Equation (4), which describe the EXIT

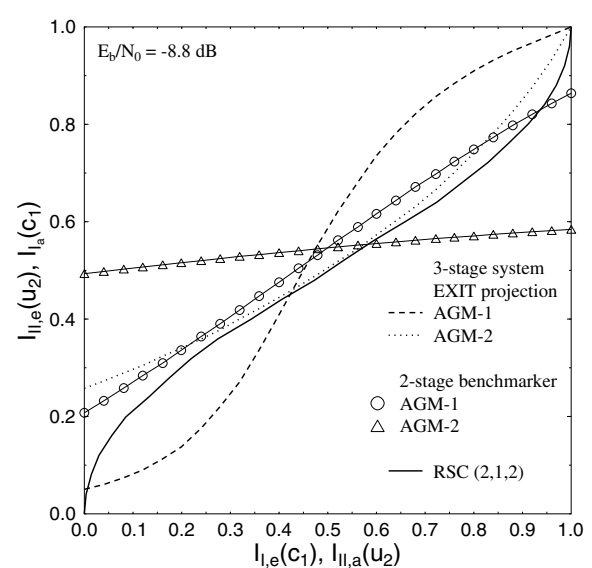

Fig. 4. Comparison of the EXIT curves for the two-stage bench-marker scheme and the 2D projection of the EXIT charts of the three-stage RSC-coded LSSTC-SP scheme, when employing the SP in conjunction with $L=16$ and using two different anti-Gray mappings AGM-1 and AGM-2, while using an interleaver length of $D=180,000$ bits.

relation between the SP demapper and Decoder II. Similarly, the 3D EXIT chart of Figure 3 can be used to describe the EXIT relation between Decoder II and Decoder I by plotting Equation (3) and Equation (5).

\section{B. 2D EXIT Chart Projection}

In this section we derive the unique and unambiguous $2 \mathrm{D}$ representations of the 3D EXIT charts of Figures 2 and 3, which may be interpreted more readily. The intersection of the surfaces in Figure 2, shown as a thick solid line, portrays the best achievable performance, when exchanging mutual information between the SP demapper and the URC Decoder II for different fixed values of $I_{I I, a}\left(\mathbf{u}_{2}\right)$ spanning the range of $[0,1]$. Each point $\left[I_{I I, a}\left(\mathbf{u}_{2}\right), I_{I I, a}\left(\mathbf{c}_{2}\right), I_{I I, e}\left(\mathbf{c}_{2}\right)\right]$ belonging to the intersection line of Figure 2 uniquely specifies a $3 \mathrm{D}$ point $\left[I_{I I, a}\left(\mathbf{u}_{2}\right), I_{I I, a}\left(\mathbf{c}_{2}\right), I_{I I, e}\left(\mathbf{u}_{2}\right)\right]$ in Figure 3 , according to the EXIT function of Equation (3). Therefore, the line corresponding to the $\left[I_{I I, a}\left(\mathbf{u}_{2}\right), I_{I I, a}\left(\mathbf{c}_{2}\right), I_{I I, e}\left(\mathbf{c}_{2}\right)\right]$ points along the thick line of Figure 2 is projected to the solid line shown in Figure 3. This projected EXIT curve may be written as

$$
I_{I I, e}\left(\mathbf{u}_{2}\right)=T_{I I, u_{2}}^{p}\left[I_{I I, a}\left(\mathbf{u}_{2}\right), E_{b} / N_{0}\right] .
$$

In Figure 4 we present the EXIT charts recorded at $E_{b} / N_{0}=$ $-8.8 \mathrm{~dB}$ for both the proposed three-stage system and of its two-stage bench-marker employing iterative detection between the SP demapper and the outer RSC decoder. The figure presents the EXIT curves for two different Anti-Gray Mapping ${ }^{2}$ (AGM) aided SP modulation schemes referred to as AGM-1 and AGM-2. The system employs a 1/2-rate memory-1 RSC code as the outer code. According to Figure 4 the intersection point between the EXIT curves of the SP demapper and the outer decoder in the two-stage bench-marker scheme does not reach the $(1.0,1.0)$ point for either of the AGM schemes due to the non-recursive nature of the SP demapper. By contrast, we observe for the three-stage system that the EXIT curves of the outer code and the 2D projection intersect at the $(1.0,1.0)$ point, resulting in an infinitesimally low BER, rather than forming an error floor as seen for the two-stage bench-marker. Notice also in Figure 4 that an open tunnel exists for the two-stage system

${ }^{2}$ Anti-Gray Mapping is used here to refer to any non-Gray mapping scheme 


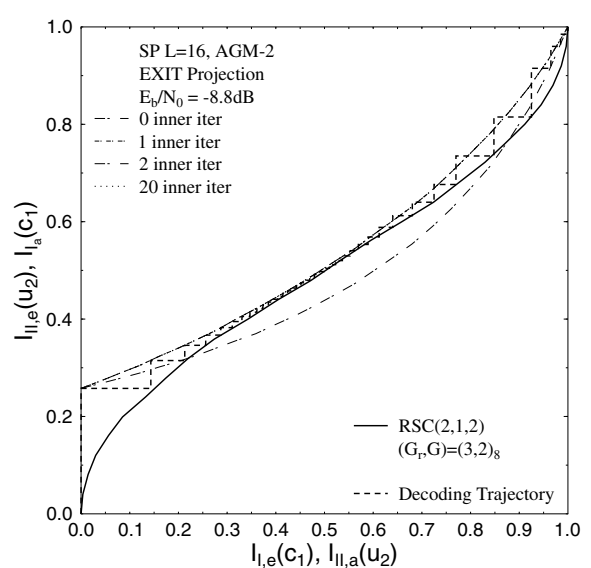

Fig. 5. 2D projection of the EXIT charts of the three-stage RSC-coded LSSTC-SP scheme, when employing the AGM-2 aided SP in conjunction with $L=16$, while using an interleaver length of $D=180,000$ bits.

employing AGM-1 at $E_{b} / N_{0}=-8.8 \mathrm{~dB}$, while an open tunnel tunnel is formed for the AGM-2 aided system at $E_{b} / N_{0}<-8.8 \mathrm{~dB}$. However, observe that the EXIT curve intersection point appears closer to the point of convergence at $(1.0,1.0)$ for the AGM-1 aided system than for its AGM-2 assisted counterpart and thus the AGM-1 aided system converges at a higher $E_{b} / N_{0}$ value than the AGM-2 assisted system, but it converges to a lower BER value. The twostage systems employing AGM-1 and AGM-2 will have an error floor. On the other hand, the three-stage system employing AGM2 exhibits an open tunnel at $E_{b} / N_{0}=-8.8 \mathrm{~dB}$ compared to the AGM-1 scheme that requires an $E_{b} / N_{0}>-8.8 \mathrm{~dB}$ for attaining convergence. Therefore, in what follows AGM-2 will be considered in the context of our three-stage system. Also, note that in the 3D EXIT curves of Figures 2 and 3 AGM-2 was employed.

Figure 5 shows the 2D-projected EXIT curve of the combined SP demapper and of the unity-rate Decoder II at $E_{b} / N_{0}=-8.8 \mathrm{~dB}$, when employing the AGM-2 scheme. Figure 5 records the 2Dprojected EXIT curves for a variable number of inner iterations carried out between the SP demapper and Decoder II. According to Figure 5, when no inner iterations are activated between the SP demapper and the URC decoder, the system requires $E_{b} / N_{0}>$ $-8.8 \mathrm{~dB}$ for maintaining an open EXIT tunnel. However, observe in Figure 5 that when 1,2 and 20 inner iterations are carried out, then an open EXIT tunnel is formed at $E_{b} / N_{0}=-8.8 \mathrm{~dB}$. Therefore, from now on we advocate using a single inner iteration that produces the same result and imposes the lowest complexity. This implies that according to the predictions of the 2D EXIT chart seen in Figure 5, the iterative decoding process is expected to converge to the $(1.0,1.0)$ point and hence an infinitesimally low BER may be attained beyond $E_{b} / N_{0}=-8.8 \mathrm{~dB}$. This expectation is confirmed by the decoding trajectory of Figure 5, which was recorded for an interleaver depth of $D=180,000$ bits.

It was argued in $[22,23]$ that the maximum achievable bandwidth efficiency of the system is proportional to the code-rate, which is equal to the area under the EXIT curve of the inner code, provided that the bit stream $\mathbf{b}$ has independently and uniformly distributed bits as well as assuming that the channel is the binary erasure channel, that the inner code rate is 1 and the MAP algorithm is used for decoding. There is also experimental evidence that the area under the inner code's EXIT curve approximates the code-rate well for

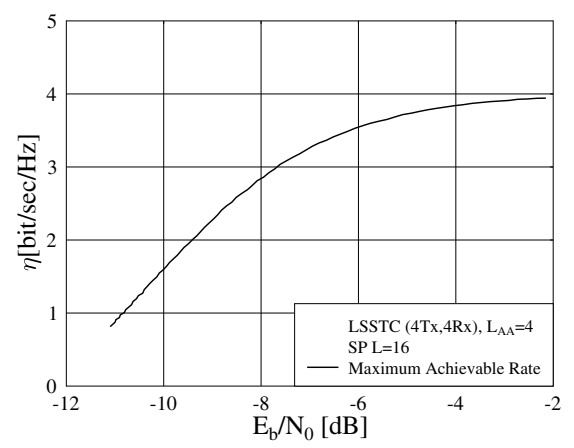

Fig. 6. Maximum Achievable rate of the LSSTC-SP scheme while employing $N_{t}=4, N_{r}=4$ and $L_{A A}=4$.

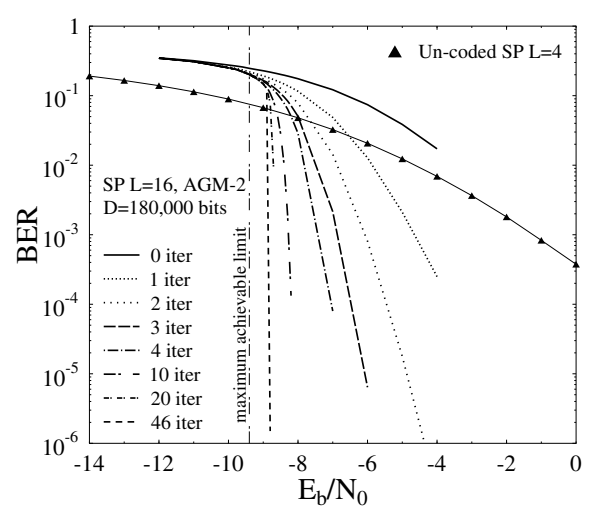

Fig. 7. BER performance of the proposed LSSTC-SP aided system employing iterative detection between a 1/2-rate RSC decoder, a URC decoder, and the SP demapper, while using an interleaver length of $D=180,000$ bits for a variable number of iterations.

both Rayleigh and Gaussian channels. Assuming that the area under the 2D projected EXIT curve of the inner decoder, i.e. the URC's decoder and the SP demapper, is represented by $\mathcal{A}$, the maximum attainable rate for the outer code is given by $R_{\max }=\mathcal{A}$ [22] at a specific $E_{b} / N_{0}$ value. Therefore, the maximum achievable bandwidth efficiency becomes $\eta_{\max }=B_{s p} \times R_{\max }$. At a bandwidth efficiency of $\eta=2 \mathrm{bit} / \mathrm{sec} / \mathrm{Hz}$, the maximum achievable rate limits inferred from Figure 6 for the LSSTC-SP scheme employing $N_{t}=4$ transmit antennas, $N_{r}=4$ receive antennas and $L_{A A}=4$ elements per AA is $E_{b} / N_{0} \approx-9.4 \mathrm{~dB}$.

\section{Performance Results}

In this section we present the performance results of the proposed multi-functional MIMO aided multi-dimensional modulation employing iterative detection, where a $1 / 2$-rate memory- 1 RSC code was employed as our outer code in conjunction with the octally represented generator polynomial $\left(G_{r}, G\right)=(3,2)$, with $G_{r}$ being the feedback generator and $G$ representing the feedforward generator. The results presented in this section correspond to a system employing an $\left(N_{t} \times N_{r}\right)=(4 \times 4)$-dimensional LSSTC-SP scheme in conjunction with $L_{A A}=4$ elements per AA.

Figure 7 compares the achievable performance of the proposed three-stage iteratively detected system employing the LSSTC-SP scheme in conjunction with $L=16$ and AGM-2 for different number of iterations against that of an uncoded LSSTC-SP scheme using $L=$ 4 , which has an identical bandwidth efficiency of $2 \mathrm{bits} / \mathrm{sec} / \mathrm{Hz}$. The figure plots the performance of the system employing an interleaver depth of $D=180,000$ bits. Explicitly, Figure 7 demonstrates that a coding advantage of about $16.5 \mathrm{~dB}$ was achieved at a BER of 


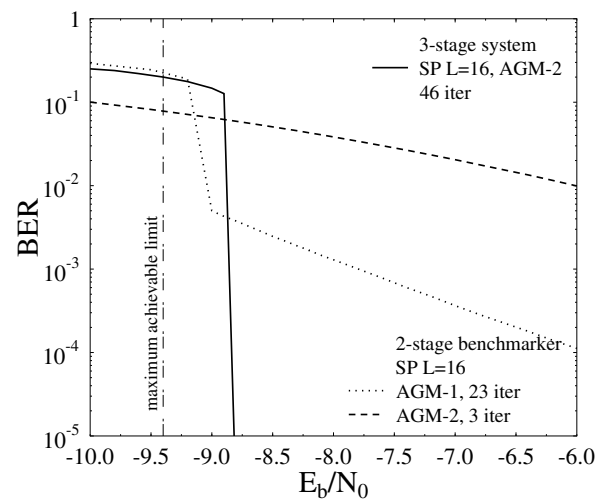

Fig. 8. BER performance of the proposed LSSTC-SP aided system employing iterative detection between a 1/2-rate RSC decoder, a URC decoder, and the SP demapper, while using an interleaver length of $D=180,000$ bits for a variable number of iterations.

$10^{-6}$ after $I=46$ iterations by the three-stage iteratively detected system over the equivalent-throughput uncoded LSSTC-SP scheme employing $L=4$. Furthermore, Figure 7 demonstrates that the BER performance closely matches the EXIT chart based prediction of Figure 5, where the system approaches an infinitesimally low BER at $E_{b} / N_{0}=-8.8 \mathrm{~dB}$ after $I=46$ iterations. We note that according to Figure 7, the proposed three-stage system performs within $0.6 \mathrm{~dB}$ from the maximum achievable rate limit of Figure 6.

Finally, Figure 8 compares the attainable performance of the threestage iteratively detected LSSTC-SP scheme in conjunction with AGM-2 in comparison to its two-stage LSSTC-SP counterpart in conjunction with AGM-1 and AGM-2. As seen in Figure 8 and as predicted in the EXIT chart of Figure 4, the three-stage iteratively detected system converges to an infinitesimally low BER at $E_{b} / N_{0}=$ $-8.8 \mathrm{~dB}$, where no BER floor is observed, which is in contrast to its two-stage iteratively detected bench-marker scheme. Observe that the two-stage iteratively detected LSSTC-SP scheme using AGM-1 employs $I=23$ iterations, while the AGM-2 aided two-stage system employs only $I=3$ iterations, since the advantage of employing any further iterations diminishes owing to the presence of a BER floor. Explicitly, the three-stage iteratively detected LSSTC-SP scheme using AGM-2 outperforms its two-stage counterpart employing AGM-1 by $4.8 \mathrm{~dB}$ and its two-stage counterpart employing AGM-2 by $9.8 \mathrm{~dB}$ at $\mathrm{BER}=10^{-5}$.

\section{CONCLUSION}

In this contribution we presented an iteratively detected threestage multi-functional MIMO aided multi-dimensional sphere packing modulation scheme. The resultant system is characterised by a high diversity gain, high multiplexing gain, beamformer gain as well as exhibiting the maximum coding advantage. Hence the system becomes capable of achieving infinitesimally low BER values, where the attainable performance is not limited by a BER floor, which is routinely encountered in conventional two-stage systems. The convergence behaviour of the three-stage system was analysed with the aid of novel 3D EXIT charts and their 2D projections. The maximum achievable rate of the system was quantified using a novel method based on EXIT charts. Explicitly, the system is capable of operating within $0.6 \mathrm{~dB}$ from the maximum achievable rate limit and of attaining at least $4.8 \mathrm{~dB}$ gain at a BER of $10^{-5}$ over the conventional iterative-detection aided two-stage bench marker scheme, where extrinsic information is exchanged between the SP demapper and the outer RSC code's decoder.

\section{REFERENCES}

[1] G. Foschini and M. Gans, "On Limits of wireless communications in a fading environment when using multiple antennas," Kluwer Academic Publishers, Wireless Personal Communications, pp. 311-335, 1998.

[2] E. Telatar, "Capacity of multi-antenna Gaussian channels," European Transactions on Telecommunications, vol. 10, pp. 585-595, Nov./Dec. 1999.

[3] P. Wolniansky, G. Foschini, G. Golden, and R. Valenzuela, "V-BLAST: an architecture for realizing very high data rates over the rich-scattering wireless channel," in International Symposium on Signals, Systems, and Electronics, (Pisa), pp. 295-300, September 1998.

[4] S. Alamouti, "A simple transmit diversity technique for wireless communications," IEEE Journal on Selected Areas in Communications, vol. 16, no. 8, pp. 1451-1458, 1998.

[5] V. Tarokh, H. Jafarkhani, and A. Calderbank, "Space-time block codes from orthogonal designs," IEEE Transactions on Information Theory, vol. 45, no. 5, pp. 1456-1467, 1999.

[6] V. Tarokh, A. Naguib, N. Seshadri, and A. Calderbank, "Combined array processing and space-time coding," IEEE Transactions on Information Theory, vol. 45, no. 4, pp. 1121-1128, 1999.

[7] M. Tao and R. Cheng, "Generalized layered space-time codes for high data rate wireless communications," IEEE Transactions on Wireless Communications, vol. 3, no. 4, pp. 1067-1075, 2004.

[8] J. Blogh and L. Hanzo, Third-generation systems and intelligent wireless networking: smart antennas and adaptive modulation. John Wiley \& Sons - IEEE Press, 2002.

[9] G. Jongren, M. Skoglund, and B. Ottersten, "Combining beamforming and orthogonal space-time block coding," IEEE Transactions on Information Theory, vol. 48, pp. 611-627, Mar. 2002.

[10] M. El-Hajjar and L. Hanzo, "Layered steered space-time codes and their capacity," Electronics Letters, vol. 43, pp. 680-682, June 2007.

[11] W. Su, Z. Safar, and K. Liu, "Space-time signal design for timecorrelated Rayleigh fading channels," in IEEE International Conference on communications, vol. 5, pp. 3175-3179, 2003.

[12] O. R. Alamri, B. L. Yeap, and L. Hanzo, "A turbo detection and spherepacking-modulation-aided space-time coding scheme," IEEE Transactions on Vehicular Technology, vol. 56, pp. 575-582, March 2007.

[13] C. Berrou, A. Glavieux, and P. Thitimajshima, "Near Shannon limit error-correcting coding and decoding: Turbo-codes. 1," in IEEE International Conference on Communications, vol. 2, (Geneva), pp. 1064-1070, May 1993.

[14] S. ten Brink, J. Speidel, and R.-H. Yan, "Iterative demapping and decoding for multilevel modulation," in IEEE Global Telecommunications Conference (GLOBCOM), vol. 1, (Sydney,NSW), pp. 579-584, 1998.

[15] S. Benedetto, D. Divsalar, G. Montorsi, and F. Pollara, "Serial concatenation of interleaved codes: performance analysis, design, and iterative decoding," IEEE Transactions on Information Theory, vol. 44, pp. 909926, May 1998.

[16] D. Divsalar, S. Dolinar, and F. Pollara, "Serial concatenated trellis coded modulation with rate-1 inner code," in IEEE Global Telecommunications Conference (GLOBECOM), vol. 2, (San Francisco, CA), pp. 777-782, 2000.

[17] S. ten Brink, "Designing iterative decoding schemes with the extrinsic information transfer chart," AË̈ International Journal of Electronics and Communications, vol. 54, pp. 389-398, Nov 2000.

[18] S. ten Brink, "Convergence of multidimensional iterative decoding schemes," in Conference Record of the Thirty-Fifth Asilomar Conference on Signals, Systems and Computers, vol. 1, (Pacific Grove, CA, USA), pp. 270-274, 2001.

[19] M. Tüchler, "Convergence prediction for iterative decoding of threefold concatenated systems," in IEEE Global Telecommunications Conference (GLOBECOM ), vol. 2, pp. 1358-1362, Nov 2002.

[20] F. Brännström, L. Rasmussen, and A. Grant, "Convergence analysis and optimal scheduling for multiple concatenated codes," IEEE Transactions on Information Theory, vol. 51, no. 9, pp. 3354-3364, 2005.

[21] J. H. Conway and N. J. Sloane, Sphere Packings, Lattices and Groups. Springer-Verlag, 1999.

[22] M. Tüchler, "Design of serially concatenated systems depending on the block length," IEEE Transactions on Communications, vol. 52, no. 2, pp. 209-218, 2004.

[23] A. Ashikhmin, G. Kramer, and S. ten Brink, "Extrinsic information transfer functions: model and erasure channel properties," IEEE Transactions on Information Theory, vol. 50, no. 11, pp. 2657-2673, 2004. 